\title{
Editorial
}

\section{Theoretical Analysis and Computational Simulation of Advanced Structured Materials}

\author{
Hanxing Zhu, ${ }^{1}$ Daniel Balint, ${ }^{2}$ and Zaoyang Guo $^{3}$ \\ ${ }^{1}$ School of Engineering, Cardiff University, Cardiff CF24 3AA, UK \\ ${ }^{2}$ Department of Mechanical Engineering, Imperial College London, South Kensington Campus, London SW7 2AZ, UK \\ ${ }^{3}$ Department of Engineering Mechanics, Chongqing University, Chongqing 400044, China \\ Correspondence should be addressed to Hanxing Zhu; zhuh3@cf.ac.uk
}

Received 11 August 2014; Accepted 11 August 2014; Published 18 December 2014

Copyright (c) 2014 Hanxing Zhu et al. This is an open access article distributed under the Creative Commons Attribution License, which permits unrestricted use, distribution, and reproduction in any medium, provided the original work is properly cited.

Structured materials may be broadly classified as comprising a single phase with an underlying topology or multiple phases in a mixture [1]. Examples include porous materials [2-5], multilayers [6,7], and functionally graded [7-9] and composite materials [10]. Structures can vary in size, typically from macro- [2] down to meso-, micro-, and nanoscales [3, 11-13], and may also be hierarchical $[12,13]$. Some structured materials comprise both topology and multiple phase mixtures at different length scales $[3,10]$, for example, metal matrix composite foams. In all cases, advanced structured materials are designed to have exceptional properties $[7-9,12,13]$ that cannot be achieved by conventional materials, for example, very high toughness, specific stiffness, multifunction $[3,4]$, and so forth. The knowledge and understanding of these supermaterials rely not only on advanced characterization and processing techniques, but also on progress in theoretical analyses and computational simulations.

Experimental measurements are truly important in developing, characterizing, and understanding advanced materials. However, they are usually very expensive and time consuming. As the material properties and performance usually depend on the interplay of many parameters and factors, it is quite difficult to understand how each of them affects the properties and performance and which one is most important. Consequently, it is difficult to obtain the global picture about how each of the parameters and factors affects the material performance only from experimental measurements. With the advancement of computer hardware and computational software, simulations (i.e., numerical experiments) play an increasingly important role in scientific research of advanced materials and structures. Compared to real experimental measurements, computer simulations are not only faster, but also more economic. Moreover, computer simulations could help us understand in detail how each of the parameters and factors affects the properties and performance of advanced materials and hence which of these are most important.

The same applies to the design of advanced materials. For example, theoretical analyses and computational simulations could guide the design of mechanical and geometrical properties of nanostructured hierarchical honeycombs and open-cell foams so that they are size dependent, tunable, and controllable $[3,12,13]$. Multilayer materials [6-9] can be designed to have extremely good thermal resistance, in addition to good mechanical strength, stiffness, and ductility. It may also be possible to design and synthesize biological materials that have mechanical properties similar to those of natural materials $[5,10]$.

This special issue focuses mainly on theoretical analyses and computational simulations of advanced structured materials including high-performance cements, shape memory alloys, nanostructured composites, and multilayered materials. We hope that readers will find in this special issue not only accurate data/results and up-to-date reviews on the relevant research areas, but also useful guidance on important areas for further research work.

Hanxing Zhu Daniel Balint Zaoyang Guo 


\section{References}

[1] D. S. Balint, V. S. Deshpande, A. Needleman, and E. van der Giessen, "Discrete dislocation plasticity analysis of the grain size dependence of the flow strength of polycrystals," International Journal of Plasticity, vol. 24, no. 12, pp. 2149-2172, 2008.

[2] H. X. Zhu, J. F. Knott, and N. J. Mills, "Analysis of the elastic properties of open-cell foams with tetrakaidecahedral cells," Journal of the Mechanics and Physics of Solids, vol. 45, no. 3, pp. 319-343, 1997.

[3] H. X. Zhu, "Size-dependent elastic properties of micro- and nano-honeycombs," Journal of the Mechanics and Physics of Solids, vol. 58, no. 5, pp. 696-709, 2010.

[4] Z. y. Guo and F. C. Caner, "Mechanical behaviour of transversely isotropic porous neo-Hookean solids," International Journal of Applied Mechanics, vol. 2, no. 1, pp. 11-39, 2010.

[5] Z. Guo, F. Caner, X. Peng, and B. Moran, "On constitutive modelling of porous neo-Hookean composites," Journal of the Mechanics and Physics of Solids, vol. 56, no. 6, pp. 2338-2357, 2008.

[6] D. S. Balint and J. W. Hutchinson, "Undulation instability of a compressed elastic film on a nonlinear creeping substrate," Acta Materialia, vol. 51, no. 13, pp. 3965-3983, 2003.

[7] H. X. Zhu, N. A. Fleck, A. C. F. Cocks, and A. G. Evans, "Numerical simulations of crack formation from pegs in thermal barrier systems with NiCoCrAlY bond coats," Materials Science and Engineering A, vol. 404, no. 1-2, pp. 26-32, 2005.

[8] D. S. Balint and J. W. Hutchinson, "An analytical model of rumpling in thermal barrier coatings," Journal of the Mechanics and Physics of Solids, vol. 53, no. 4, pp. 949-973, 2005.

[9] D. S. Balint, S.-S. Kim, Y. F. Liu, R. Kitazawa, Y. Kagawa, and A. G. Evans, "Anisotropic TGO rumpling in EB-PVD thermal barrier coatings under in-phase thermomechanical loading," Acta Materialia, vol. 59, no. 6, pp. 2544-2555, 2011.

[10] Z. Y. Guo, X. Q. Peng, and B. Moran, "A composites-based hyperelastic constitutive model for soft tissue with application to the human annulus fibrosus," Journal of the Mechanics and Physics of Solids, vol. 54, no. 9, pp. 1952-1971, 2006.

[11] Z. P. Bažant and Z. Guo, "Size effect and asymptotic matching approximations in strain-gradient theories of micro-scale plasticity," International Journal of Solids and Structures, vol. 39, no. 21-22, pp. 5633-5657, 2002.

[12] H. X. Zhu, L. B. Yan, R. Zhang, and X. M. Qiu, "Size-dependent and tunable elastic properties of hierarchical honeycombs with regular square and equilateral triangular cells," Acta Materialia, vol. 60, no. 12, pp. 4927-4939, 2012.

[13] H. X. Zhu and Z. B. Wang, "Size-dependent and tunable elastic and geometric properties of hierarchical nano-porous materials," Science of Advanced Materials, vol. 5, no. 6, pp. 677686, 2013. 

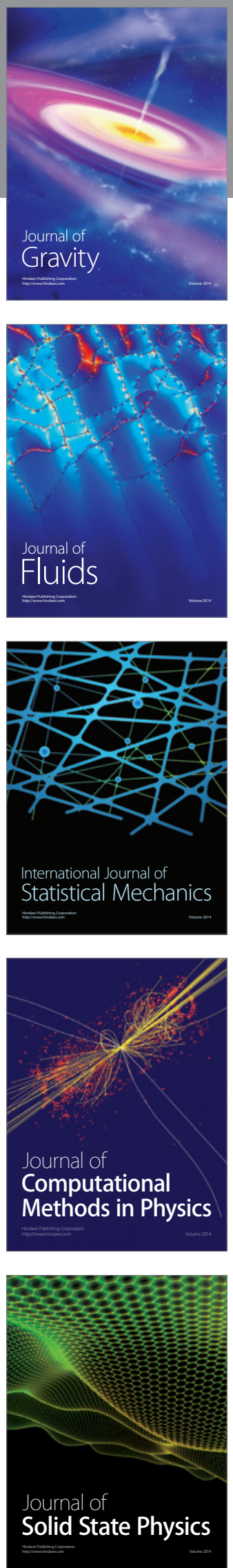

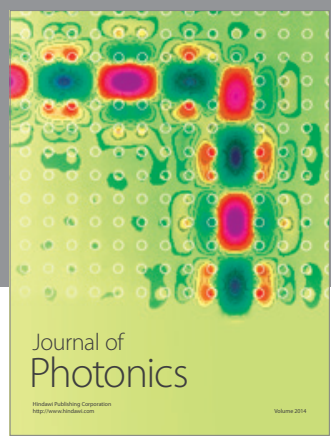

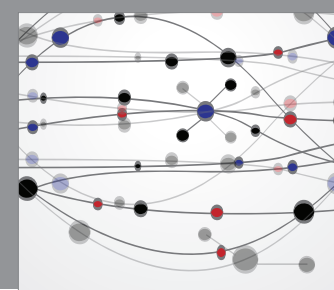

The Scientific World Journal

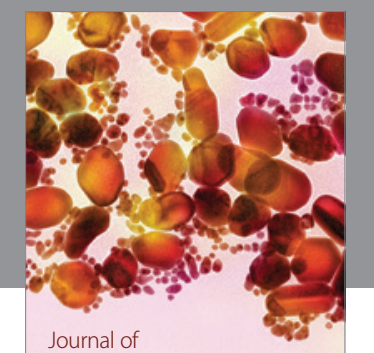

Soft Matter
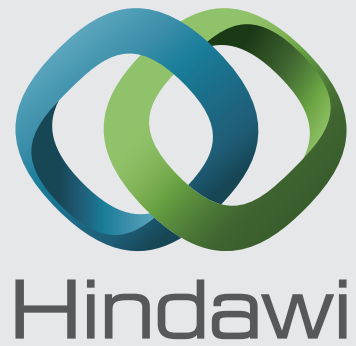

Submit your manuscripts at

http://www.hindawi.com
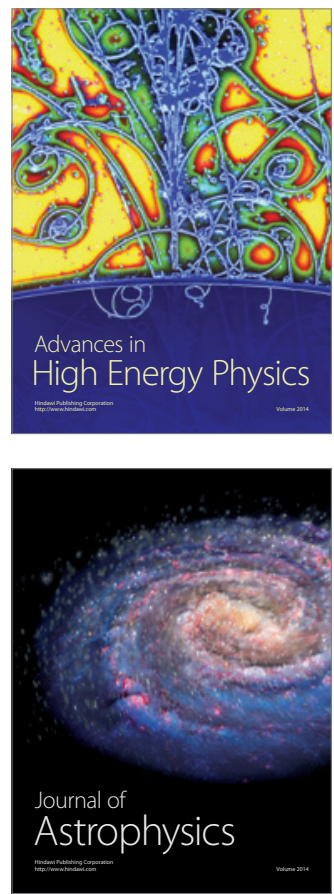
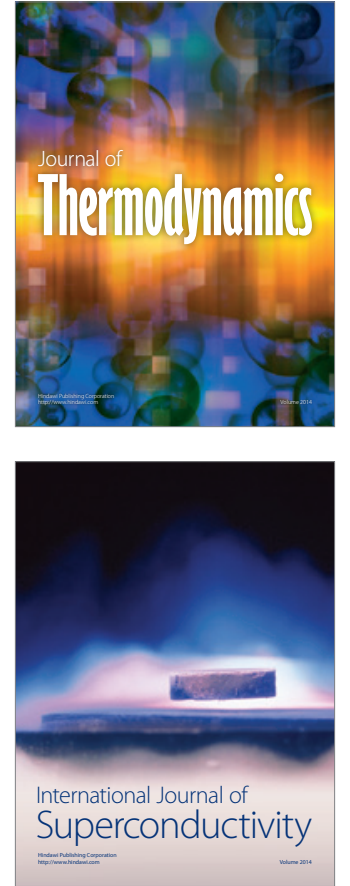
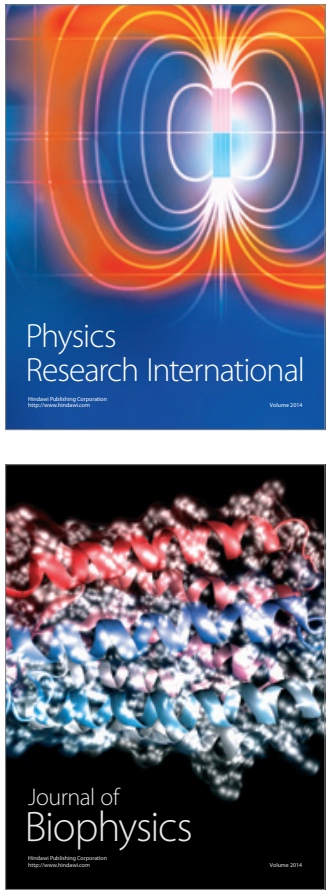
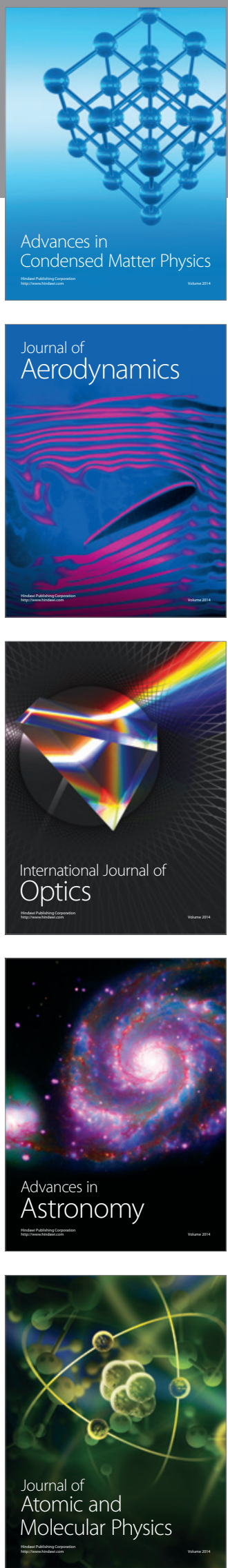